\title{
ESTUDIO DE FACTIBILIDAD TÉCNICA Y ECONÓMICA ORIENTADO A LA UTILIZACIÓN DE RESIDUOS DE TIERRA DIATOMACEA Y LODO DE CAL RESULTANTES DE LA INDUSTRIA CERVECERA EN LA FABRICACIÓN DE BLOQUES DE GRES.
}

\author{
DIRECTOR \\ JORGE SÁNCHEZ MOLINA \\ INGENIERO QUÍMICO \\ VICERRECTOR DE INVESTIGACIÓN Y \\ EXTENSIÓN \\ INVESTIGADORES \\ JAZMÍN DURÁN ANGARITA \\ YADIR XIOMARA PINTO GELVEZ \\ INGENIERAS DE PRODUCCIÓN \\ INDUSTRIAL \\ UNIVERSIDAD FRANCISCO DE PAULA \\ SANTANDER
}

\section{INTRODUCCIÓN}

En las últimas décadas la producción industrial ha causado un aumento en la generación de residuos, que comúnmente se disponen en las fuentes naturales sin ningún tipo de tratamiento y sin tener en cuenta la capacidad limitada de estas fuentes, afectando significativamente el equilibrio natural del ambiente. Conscientes de esta problemática, se efectúa un estudio a nivel de laboratorio e industrial con miras a la utilización de residuos del proceso cervecero, como son la cal y la tierra diatomácea. El proyecto busca evaluar la factibilidad técnica y económica de fabricar bloques de gres con cal y/o tierra diatomácea, como alternativa de solución enfocada hacia la problemática de los residuos. La aceptación de usar el residuo de cal o tierra diatomácea de la industria cervecera, está sujeta a diferentes aspectos dentro de los que se encuentra: el mejoramiento de las especificaciones técnicas de los bloques, el costo de producción, la inversión requerida y la contribu- ción en la conservación del ambiente como uno de los aspectos más relevantes del proyecto.

\section{PROCEDIMIENTO}

\section{Diseño de experimentos pre- liminares}

Para los experimentos preliminares se realizaron mezclas de arcilla con contenido de $0,5,10$, y $15 \%$ en peso de residuos de cal y tierra diatomácea, por considerarse estos valores suficientes para observar una tendencia en el comportamiento de las propiedades a evaluar. A escala de laboratorio se realizaron 11 mezclas en total: tres de arcilla con tierra diatomácea, tres de arcilla con cal y cinco mezclando arciIla, tierra diatomáceas y cal en las diferentes proporciones. Además se realizaron bloques de la mezcla actualmente utilizada, con el fin de tener un parámetro de comparación. A los bloques se le efectuaron análisis cualitativos y cuantitativos.

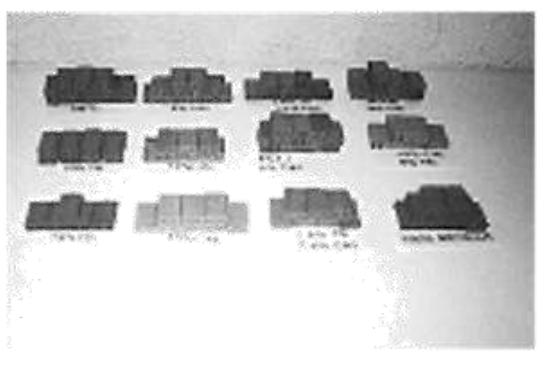

\subsection{Preparación de muestras a escala de laboratorio}

Se elaboraron 20 bloques a escala 1:10 respecto a las dimensiones del bloque usadas comercialmente. De estos bloques, se utilizaron cinco unidades para determinar cada propiedad de resistencia a la compresión en crudo, resistencia a la compresión en seco, ensayos de absorción y análisis cualitativo. Los ensayos de las piezas crudas y cocidas se realizaron en el laboratorio de Resistencia de Materiales de la Universidad Francisco de Paula Santander, (también a escala industrial). En el análisis cualitativo se observaron el área superficial, la erosionabilidad, laminaciones, grietas y alabeo o curvatura. En los análisis cuantitativos se determinaron la resistencia a la compresión y la absorción de agua de las piezas cocidas, acorde con las Normas Técnicas Colombianas, ICONTEC. Los ensayos de absorción se realizaron en el Laboratorio de Suelos Civiles de la Universidad Francisco de Paula Santander (A escala de laboratorio e industrial).

Mediante ensayos a nivel de laboratorio se determinaron las condiciones para elaborar un producto cerámico, que cumpliese con las especificaciones mínimas para su uso en construcción, y que además, conserve o mejore sus características.

Las exigencias mínimas establecidas por las Normas Técnicas Colombianas, ICONTEC, para este tipo de productos utilizados en la industria de la construcción, se refieren principalmente a resistencia a la compresión y absorción de agua. La NTC 4017 establece los procedimientos de muestreo y 
ensayo de resistencia mecánica a la compresión y absorción de agua de unidades de mampostería de arcilla. La NTC 4205 establece los valores permitidos de resistencia a la compresión y de absorción de agua de unidades mampuestas para construcción.

\subsubsection{Análisis Cualitativo mediante inspección visual}

Durante la elaboración de los ensayos a nivel de laboratorio se observó que los bloques de las mezclas tres y seis del cuadro No.1 presentan desperfectos al salir de la extrusora, generando perdida de tiempo en la reelaboración y desperdicio del material. Realizado el proceso de cocción de los bloques se observó que la mezcla tres presenta área superficial regular, grietas y laminaciones; la mezcla cuatro presenta alabeo; en la mezcla nueve se observa área superficial regular y grietas; la mezcla diez presenta área superficial regular, laminaciones y alabeo y por último la mezcla once presenta área superficial regular y laminaciones. Las demás mezclas cumplen las especificaciones mínimas de apariencia.

\subsubsection{Análisis Cuantitativo}

Después de la cocción, a los bloques se le analizaron las siguientes características y se concluyó:

Masa. En las mezclas de tierra diatomáceas y arcilla a medida que se aumenta el porcentaje de residuo
Tabla No.1

\begin{tabular}{|l|l|l|l|l|l|l|}
\hline $\begin{array}{l}\# \\
\text { Mezc }\end{array}$ & Mezcla & $\begin{array}{l}\text { Área } \\
\text { superfici }\end{array}$ & Erosionabi & Laminac & Grieta & $\begin{array}{l}\text { Alabeo } \\
\text { curvatu }\end{array}$ \\
\hline 1 & $5 \% \mathrm{TD}$ & Buena & Buena & No & No & No \\
\hline 2 & $10 \% \mathrm{TD}$ & Buena & Buena & No & No & No \\
\hline 3 & $15 \% \mathrm{TD}$ & Regular & Buena & $\mathrm{Si}$ & $\mathrm{Si}$ & No \\
\hline 4 & $5 \% \mathrm{CAL}$ & Buena & Buena & No & No & Si \\
\hline 5 & $10 \% \mathrm{CAL}$ & Buena & Buena & No & No & No \\
\hline 6 & $15 \% \mathrm{CAL}$ & Buena & Buena & No & No & No \\
\hline 7 & $\begin{array}{l}2.5 \% \mathrm{TD} \\
2.5 \% \mathrm{CAL}\end{array}$ & Buena & Buena & No & No & No \\
\hline 8 & $\begin{array}{l}5 \% \mathrm{TD} \\
5 \% \mathrm{CAL}\end{array}$ & Buena & Buena & No & No & No \\
\hline 9 & $\begin{array}{l}7.5 \% \mathrm{TD} \\
7.5 \% \mathrm{CAL}\end{array}$ & Regular & Buena & No & Si & No \\
\hline 10 & $\begin{array}{l}10 \% \mathrm{TD} \\
5 \% \mathrm{CAL}\end{array}$ & Regular & Buena & $\mathrm{Si}$ & No & Si \\
\hline 11 & $\begin{array}{l}5 \% \mathrm{TD} \\
10 \% \mathrm{CAL}\end{array}$ & Regular & Buena & $\mathrm{Si}$ & No & No \\
\hline 12 & $100 \% \mathrm{ARC}$ & Buena & Buena & No & No & No \\
\hline
\end{tabular}

Mezclas optimas en el análisis cualitativo

Tabla No.2

\begin{tabular}{|c|c|c|c|c|c|c|}
\hline $\begin{array}{c}\# \\
\text { Mezcla }\end{array}$ & Mezcla & $\begin{array}{c}\text { Masas } \\
\text { de } \\
\text { piezas } \\
\text { cocidas } \\
\text { grs. }\end{array}$ & $\begin{array}{c}\text { \% } \\
\text { Humed }\end{array}$ & $\begin{array}{c}\text { Resistencia a } \\
\text { la compresión } \\
\text { Crudo } \\
\text { (Kgf/cm) }\end{array}$ & $\begin{array}{c}\text { Resistencia a } \\
\text { la compresión } \\
\text { Piezas cocidas } \\
\mathbf{2} \\
\text { (Kgf/cm) }\end{array}$ & $\begin{array}{c}\% \\
\text { Absor } \\
\text { ebullic }\end{array}$ \\
\hline 1 & $5 \% \mathrm{TD}$ & 9,89 & 22,22 & 8,79 & 78,00 & 13,46 \\
\hline 2 & $10 \% \mathrm{TD}$ & 9,55 & 25,00 & 6,50 & 61,03 & 17,31 \\
\hline 3 & $15 \% \mathrm{TD}$ & 8,71 & 26,66 & 4,72 & 40,11 & 17,91 \\
\hline 4 & $5 \% \mathrm{CAL}$ & 9,88 & 16,66 & 4,29 & 58,95 & 12,36 \\
\hline 5 & $10 \% \mathrm{CAL}$ & 9,78 & 16,66 & 4,53 & 60,53 & 14,08 \\
\hline 6 & $15 \% \mathrm{CAL}$ & 9,76 & 16,66 & 9,84 & 63,54 & 15,21 \\
\hline 7 & $\begin{array}{c}2.5 \% \mathrm{TD} \\
2.5 \% \mathrm{CAL}\end{array}$ & 9,90 & 17,50 & 9,83 & 91,17 & 11,64 \\
\hline 8 & $\begin{array}{c}5 \% \mathrm{TD} \\
5 \% \mathrm{CAL}\end{array}$ & 9,81 & 21,66 & 3,39 & 62,22 & 16,20 \\
\hline 9 & $\begin{array}{c}7.5 \% \mathrm{TD} \\
7.5 \% \mathrm{CAL}\end{array}$ & 9,04 & 21,66 & 3,11 & 78,49 & 17,97 \\
\hline 10 & $\begin{array}{c}10 \% \mathrm{TD} \\
5 \% \mathrm{CAL}\end{array}$ & 11,11 & 21,66 & 3,17 & 54,97 & 19,80 \\
\hline 11 & $\begin{array}{c}5 \% \mathrm{TD} \\
10 \% \mathrm{CAL}\end{array}$ & 9,28 & 21,00 & 3,57 & 49,20 & 18,19 \\
\hline 12 & $100 \% \mathrm{ARC}$ & 10,05 & 16,66 & 3,66 & 57,75 & 10,78 \\
\hline & & & & & \\
\hline
\end{tabular}

Para obtener estos valores se eliminaron los resultados mayores y menores y se promediaron los tres restantes. 
adicionado disminuye la masa, lo cual indica que es un material con altas perdidas al calor. En los bloques realizados con residuo de cal y arcilla, la medida de la masa se mantiene en un rango de variación mínimo. Los bloques de la mezcla diez presentan la mayor masa obtenida.

Porcentaje de humedad. La cantidad de agua necesaria para hacer la pasta moldeable y no pegajosa, aumenta proporcionalmente con el porcentaje de residuo de tierra diatomáceas adicionada ya que los granos finos de ésta absorben gran cantidad de agua, causando una disminución en la manejabilidad de la mezcla. Para las mezclas con adicción de residuo de cal se mantuvo constante el porcentaje de humedad. En los bloques realizados con adicción de los tres materiales también se mantuvo un valor constante del porcentaje de humedad, excepto para la mezcla siete.

Absorción de agua. La tierra diatomáceas es un material altamente poroso, por lo tanto, produce un aumento en la absorción de agua de los bloques, el cual se debe a la mayor porosidad del bloque causado por los espacios vacíos que dejan los gases producidos por la oxidación de la materia orgánica durante la cocción.

A medida que se aumenta la adición de residuo de tierra diatomáceas, aumenta el porcentaje de absorción de agua.

A medida que se aumenta la adición de residuo de cal, aumenta el porcentaje de absorción, pero con valores inferiores a los presentados en las mezclas con tierra diatomáceas.

A medida que aumenta la adición de residuo (mezcla 7, 8, 9 de los tres materiales) se presentó un aumento en el porcentaje de absorción.

Resistencia a la compresión. Se puede afirmar que la resistencia a la compresión disminuye a medida que aumenta la adición de residuo de tierra diatomácea. La resistencia a la compresión aumenta propor-cionalmente con la adicción de residuo de cal.

Los bloques en donde se mezclaron los tres materiales, presentaron resistencia a la compresión que se encuentra entre los valores mínimos requeridos por la norma NTC 4205 , excepto la mezcla 11.

Después de realizar los análisis cualitativos y cuantitativos de las diferentes mezclas, se determinó que los bloques que presentaron las mejores características para ser elaborados a nivel industrial corresponden a la mezcla $2(10 \%$ tierra diatomácea, $90 \%$ arcilla) y la mezcla 5 (10\% cal y $90 \%$ arcilla).

\subsection{Elaboración de bloques a escala industrial}

Con base en los resultados obtenidos en el laboratorio, se realizaron dos pruebas a nivel industrial, en una se mezcló $10 \%$ de residuo de tierra diatomácea con
$90 \%$ de arcilla (lo equivalente a $450 \mathrm{Kg}$ de arcilla y $50 \mathrm{Kg}$ de residuo seco de tierra diatomácea) y en la otra $10 \%$ de residuo de cal con $90 \%$ de arcilla (450 Kg de arcilla y $50 \mathrm{Kg}$ de cal), hasta obtener un material homogéneo.

El material mezclado, se coloca en la banda transportadora que va hacia la mezcladora donde será humedecido hasta formar una pasta arcillosa; la cual se moldea por extrusión al vacío y se corta con las dimensiones establecidas comercialmente, formándose de esta manera los bloques que serán transportados a una zona cubierta para ser secados naturalmente, $y$ posteriormente se transportados al proceso de cocción y enfriamiento.

\subsubsection{Análisis cualitativo de resultados.}

Los bloques elaborados son unida-des de mampostería de perforación horizontal, no estructural, que se utilizan para muros divisorios o de cierre que únicamente atienden las cargas debido a su propio peso. Sus características comerciales son:

Dimensiones:

$30 \mathrm{~cm} \times 20 \mathrm{~cm} \times 10 \mathrm{~cm}$

Espesor de las paredes: $10 \mathrm{~mm}$

Espesor de tabiques: $6 \mathrm{~mm}$

Apariencia: Bloque vitrificado

Después de fabricar los bloques a nivel industrial con adición de residuo, éstos se sometieron a inspec- 
Tabla No.3

\begin{tabular}{|c|l|l|l|l|l|}
\hline Mezcla & $\begin{array}{c}\text { Área } \\
\text { superficial }\end{array}$ & Erosionabil & Laminación & Grietas & $\begin{array}{c}\text { Alabeo } \\
\text { curvatura }\end{array}$ \\
\hline $10 \% \mathrm{TD}$ & Buena & Buena & No & No & No \\
\hline $10 \% \mathrm{CAL}$ & Buena & Buena & No & No & No \\
\hline $100 \%$ ARCIL & Buena & Buena & No & No & No \\
\hline
\end{tabular}

Según análisis cualitativo los resultados de las dos mezclas estudiadas dieron favorables.

ción visual. Los bloques elaborados con tierra diatomáceas y cal mostraron las mismas características: color uniforme, libre de grietas, laminaciones y no presentaron alabeo. En la elaboración de los bloques con tierra diatomácea se presentó mayor dificultad, debido al olor fétido que indispuso a los operarios.

Con la adición de residuo de cal la elaboración de los bloques fue más rápida, no se presentó desperdicio de material ni de tiempo. Además el residuo de cal no presenta olor desagradable que incomode a los operarios.

\subsubsection{Análisis cuantitativo}

Masa. Al adicionar los dos residuos se disminuye la masa de los bloques en comparación con los que se fabrican actualmente. La masa promedio de los bloques con adición de residuo de tierra diatomácea es de 4,3 Kg. y los de arcilla pura $4,95 \mathrm{Kg}$.

Tabla No.4

\begin{tabular}{|c|c|c|c|c|c|c|}
\hline \multirow{2}{*}{ Mezcla } & \multirow{2}{*}{$\begin{array}{l}\text { Masa piezas } \\
\text { cocidas } \\
\mathrm{Kg} .\end{array}$} & \multicolumn{2}{|c|}{ \% Contracción } & \multirow{2}{*}{$\begin{array}{c}\text { Resistencia a } \\
\text { la compresión } \\
\text { Crudo } \\
\left(\mathrm{Kgf} / \mathrm{cm}^{2}\right)\end{array}$} & \multirow{2}{*}{$\begin{array}{c}\text { Resistencia a } \\
\text { la compresión } \\
\text { Piezas cocidas } \\
\left(\mathrm{Kgf} / \mathrm{cm}^{2}\right)\end{array}$} & \multirow{2}{*}{$\begin{array}{l}\text { \% Absorción } \\
\text { cbullición }\end{array}$} \\
\hline & & Secado & Cocción & & & \\
\hline Arcilla & 4.950 & 4,10 & 0,78 & 3,57 & 21,54 & 10,41 \\
\hline $10 \% \mathrm{TD}$ & 4.300 & 5,95 & 0,40 & 2,98 & 21,91 & 12,73 \\
\hline $10 \% \mathrm{Cal}$ & 4.650 & 4,85 & 0,78 & 2,71 & 24,49 & 13,20 \\
\hline
\end{tabular}

Resultado del análisis cuantitativo de los bloques elaborados a nivel industrial.

Presentándose una disminución de 0,6 Kg. Con adición de residuo de cal también se disminuye la masa pero en un valor menor que el presentado con adición de tierra diatomáceas.

Absorción de agua. Los resultados de laboratorio son satisfactorios, quedando los bloques con residuos de tierra diatomáceas y cal dentro de la norma establecida por la NTC 4205, aunque se aumenta la absorción en comparación con los bloques producidos actualmente.

Resistencia a la compresión. Los bloques elaborados (arcilla pura y las mezclas con residuos), se sometieron a los ensayos de resistencia a la compresión y presentaron valores inferiores a los especificados en la norma NTC 4205. Con el propósito de verificar si el proceso de vitrificación afecta la resistencia se realizan los mismos ensayos a los bloques vitrificados y no vitrificados de la competencia para obtener un parámetro de comparación entre los bloques obtenidos y los que actualmente se utilizan en la construcción.

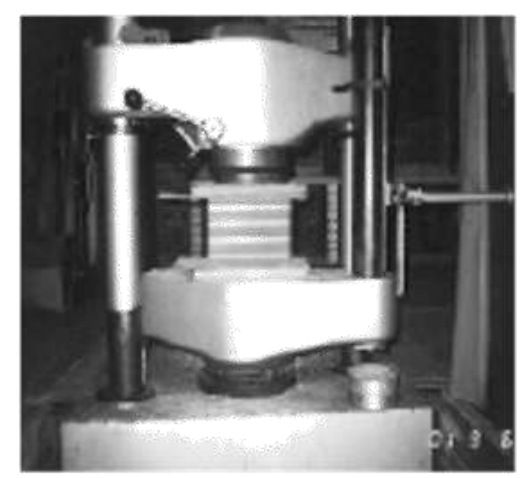

Maquina Universal de ensayos utilizada para determinar la resistencia a la compresión de las piezas.

Los bloques seleccionados de la competencia para evaluar son no estructurales, teniendo en cuenta que los elaborados para la investigación son de este tipo. Se observó que los bloques vitrificados presentaron valores menores a los exigidos por la norma. 


\subsection{Resistencia a la compresión de los bloques producidos por cuatro empresas destacadas de Norte de Santander.}

Tabla No.5

\begin{tabular}{|l|l|l|}
\hline \multicolumn{1}{|c|}{ Empresa } & $\begin{array}{l}\text { Masa } \\
\text { (Kg) }\end{array}$ & $\begin{array}{l}\text { Resistencia a la } \\
\text { compresión }\left(\mathbf{k g f} / \mathbf{c m}^{2}\right)\end{array}$ \\
\hline Tejar A (No vitrificado) & 4.608 & 37,38 \\
\hline Tejar B (No vitrificado) & 4.860 & 45,90 \\
\hline Tejar C (No vitrificado) & 5.363 & 31,00 \\
\hline Tejar D (Vitrificado) & 5.000 & 14,86 \\
\hline
\end{tabular}

Descripción de bloques producidas en las 4 principales empresas de Norte de Santander, respecto a las dos principales características comerciales.

En el análisis realizado a la competencia se determinó que la resistencia a la compresión de los bloques vitrificados es de 14,86 Kgf $/ \mathrm{cm}^{2}$. Los bloques vitrificados que actualmente se fabrican presentan una resistencia de $21,54 \mathrm{kgf} / \mathrm{cm}^{2}$.

Con adición de residuo de tierra diatomáceas los bloques presentaron resistencia a la compresión de $21,91 \mathrm{kgf} / \mathrm{cm}^{2}$ y con adición de residuo de cal la resistencia a la compresión de los bloques fue de $24,49 \mathrm{kgf} / \mathrm{cm}^{2}$. Los valores anteriores demuestran que con adición de los residuos se mejora la resistencia a la compresión de los bloques.

Finalmente se puede concluir que con adición de los residuos de tierra diatomáceas y cal se obtiene un producto de menor masa, mayor porosidad, mayor resistencia a la compresión, menor agrietamiento y de color uniforme en comparación con los bloques fabricados actualmente.

Teniendo en cuenta que los dos residuos (tierra diatomáceas y cal) afectan favorablemente las características técnicas de los bloques, se efectúa el estudio de ingeniería y la evaluación económica.

\section{CONCLUSIONES}

Como conclusiones, de acuerdo con el trabajo experimental, la evaluación técnica y la evaluación económica de las alternativas propuestas en este estudio se puede afirmar que:

-El sistema propuesto para el manejo y disposición de los residuos repre- senta una solución viable al problema ambiental y social que se presenta actualmente.

-Los residuos de tierra diatomácea y cal pueden utilizarse en la fabricación de bloques en una proporción de hasta $10 \%$, ya que los resultados de los ensayos efectuados a estas mezclas son factibles técnicamente.

-Con la adición de los residuos en la fabricación de bloques se obtiene un producto de menor masa, situación favorable al momento de exportar ya que la disminución en la masa genera disminución de costos. Además, al disminuir la masa se favorecen los costos de estructura y cimentación de las edificaciones.

-Mediante las inspecciones efec-tuadas a los bloques elaborados a nivel industrial, se determinó que no presentan alabeo ni laminaciones. Además presentan menor agrietamiento y color uniforme.

-La resistencia a la compresión es la propiedad de mayor importancia en los bloques. Con la utilización de residuos de cal y tierra diatomáceas se logra mejorar esta característica técnica lo cual le permite a la industria de la arcilla ofrecer un producto de mejor calidad a sus clientes.

-La resistencia a la compresión de los bloques se mejora en com- 
paración con los bloques fabricados actualmente, pero estos valores son menores a los establecidos por la norma NTC 4205 debido a que la vitrificación afecta esta propiedad.

-Una de las limitantes en el uso del residuo de tierra diatomáceas radica en el mal olor que éste genera. Esta situación se reflejo durante la elaboración de los bloques a nivel industrial ya que el olor del residuo indispuso al personal operativo.

-La fabricación de bloques con adición de residuos no afecta considerablemente el proceso actual, esta situación es favorable para la ejecución del proyecto, ya que se puede realizar con los recursos con que cuentan las empresas ladrilleras de la región.

-Del estudio de financiero se pudo concluir que la elaboración de bloques con adición de residuos incrementa los costos actuales, debido a que se debe cargar un nuevo concepto por transporte de los lodos desde la empresa cervecera hasta la ladrillera. Es importante aclarar que en otra empresa podría ser factible.

-La utilización de los residuos de tierra diatomácea y cal en la fabricación de bloques es factible desde el punto de vista técnico y contribuye a conservar el ambiente, pero económicamente no es viable.

\section{BIBLIOGRAFÍA}

HODSON, William. MAYNARD Manual del Ingeniero Industrial. Editorial Mc Graw-Hill, cuarta edición. México, 1996. Tomo 4. P. 15-65

ICONTEC, Instituto Colombiano de Normas Técnicas NTC 296, 1075, 1486, 1487, 2153, 4017, 4025.

KIRK, E Raymond. Enciclopedia de Tecnología Química. Editorial Hispanoamericana, México, 1965.

Tomo 3. P.10-47
Memorias de II Encuentro Nacional de Investigadores. Medellín, 1996. P. 133

Memorias III Encuentro Nacional de Arcilla y la cerámi-ca. Cúcuta 1991. P. 290

MIRANDA M. Juan José. Gestión de proyectos, Colombia editorial Guadalupe, segunda edición 1998. P. 367

PERRY Robert. Manual del Ingeniero Químico, México

McGRAW- HIL, sexta edición 1992. Tomo 1. P 12-59

Programa Cerámicas y Vítreos. Medellín, 1994. P. 120

SAPAG Nassir, SAPAG Reinaldo. Preparación y evaluación de proyectos. Editorial Mc Graw Hill, Chile 2000. P. 390

TREYBAL, Robert. Operaciones de Transferencia de masa. Editorial Mc Graw-Hill, segunda edición. 1994. P. 362. 
